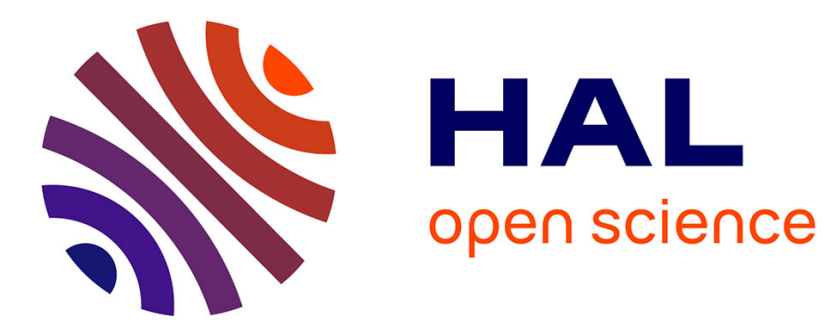

\title{
SRB: A Selective Reliable Broadcast Protocol for Safety Applications in VANETs
}

\author{
Anna Maria Vegni, Alessio Stramacci, Enrico Natalizio
}

\section{To cite this version:}

Anna Maria Vegni, Alessio Stramacci, Enrico Natalizio. SRB: A Selective Reliable Broadcast Protocol for Safety Applications in VANETs. [Research Report] 2012. hal-00705311

HAL Id: hal-00705311

https://hal.inria.fr/hal-00705311

Submitted on 7 Jun 2012

HAL is a multi-disciplinary open access archive for the deposit and dissemination of scientific research documents, whether they are published or not. The documents may come from teaching and research institutions in France or abroad, or from public or private research centers.
L'archive ouverte pluridisciplinaire HAL, est destinée au dépôt et à la diffusion de documents scientifiques de niveau recherche, publiés ou non, émanant des établissements d'enseignement et de recherche français ou étrangers, des laboratoires publics ou privés. 


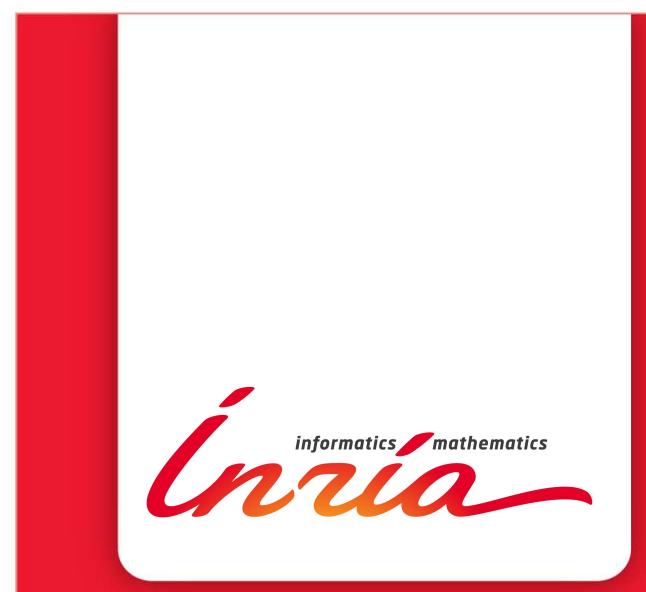

\section{SRB: A Selective Reliable Broadcast Protocol} for Safety Applications in VANETs

Anna Maria Vegni, Alessio Stramacci, Enrico Natalizio

RESEARCH 



\title{
Iñía
}

\section{$S R B$ : A Selective Reliable Broadcast Protocol for Safety Applications in VANETs}

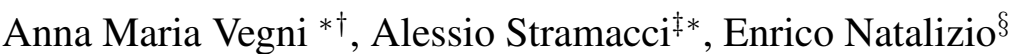 \\ Project-Teams FUN
}

Research Report $n^{\circ}$ XXXX — June 2012 - 14 pages

\begin{abstract}
In this paper we present a novel vehicular communication protocol, which aims to reduce the effect of broadcast storm problem in VANETs. When the traffic density is above a certain value (e.g., when vehicles are in congested traffic scenarios), one of the most serious problems is the increase of packet collisions and medium contentions among vehicles which attempt to communicate.

Our proposed technique, namely Selective Reliable Broadcast protocol (SRB), is intended to limit the number of packet transmissions, by means of opportunistically selecting neighboring nodes, acting as relay nodes. As a result, the number of forwarder vehicles is strongly reduced, while network performance is preserved. SRB belongs to the class of broadcast protocols, and exploits the traditional vehicular partitioning behavior to select forwarders. Each cluster is automatically detected as a zone of interest, whenever a vehicle is approaching, and packets will be forwarded only to selected vehicles, opportunistically elected as cluster-heads. In respect of traditional broadcast approaches, the main strengths of SRB are the efficiency of detecting clusters and selecting forwarders in a fast way, in order to limit the broadcast storm problem. Simulation results have been carried out both in urban and highway scenarios, in order to validate the effectiveness of SRB, in terms of cluster detection and reduction of number of selected forwarders.
\end{abstract}

Key-words: VANETs, cluster-based protocol, broadcast storm problem.

This paper appears in Proc. of Intl. Conf. on Selected Topics in Mobile \& Wireless Networking (iCOST 2012), July 2-4, 2012, Avignon, France.

* Department of Applied Electronics, University of Roma Tre, Rome, Italy.

$\dagger$ amvegni@uniroma3.it

¥ale.stramacci@stud.uniroma3.it

$\S$ FUN Research Group, INRIA Lille - Nord Europe, France, enrico.natalizio@inria.fr

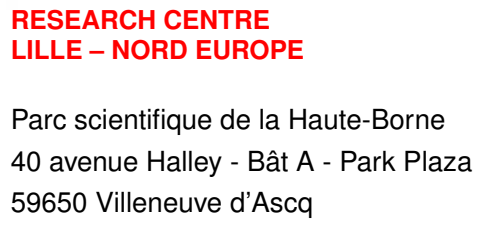


SRB: A Selective Reliable Broadcast Protocol for Safety Applications in VANETs

Résumé : Pas de résumé

Mots-clés : Pas de motclef 


\section{Introduction}

Vehicular Ad hoc NETworks (VANETs) are emerging as the preferred network design for Intelligent Transportation Systems (ITS), providing communications among nearby vehicles in the support of Internet access, as well as a variety of safety applications.

VANETs come from traditional Mobile Ad-hoc NETworks (MANETs), while showing many different aspects. They consist of mostly highly mobile nodes moving in the same or opposite directions (i.e., vehicles), forming clusters [1]. Vehicles moving along different but nearby roads are not always able to communicate each other due to connectivity disruptions caused by quick topology network changes, vehicle speed and sparse or totally disconnected scenarios.

Inter-vehicle communications are expected to significantly improve transportation safety and mobility on the road. Several applications of inter-vehicle communications have been identified, from safety and warning applications, up to traffic control and driver assistance applications [1]. Many of these applications require multicast routing protocol to a group of vehicles satisfying a geographical criterion.

In this vision, most applications targeting VANETs rely heavily on broadcast transmission both to discover nearby vehicles, and to disseminate traffic-related information to all reachable vehicles within a certain geographical area, rather than only to routing-selected hosts, like in MANETs. On the other side, broadcasting packets may lead to frequent contention and collisions in transmission among neighboring vehicles. This problem is sometimes referred to as the broadcast storm problem [2, 3]. This affects inter-vehicle communications, since redundant rebroadcasts, contention and collisions can be largely increased. When a vehicle rebroadcasts a message, it is highly likely that the neighboring vehicles have already received it, and this results in a large number of redundant messages. Although multiple solutions exist to alleviate the broadcast storm effect in the usual MANET environment, only a few solutions have been proposed addressing the VANET context [4, 5].

In this paper, we propose an effective broadcast method for safety alerts in VANETs, called as Selective Reliable Broadcast (SRB) protocol. The main aim is to reduce the broadcast storm problem, since SRB selects only one vehicle within a cluster -namely, a cluster-head-in order to efficiently rebroadcast emergency and control messages. SRB technique is able to detect the well-known car platoons, which cause traffic congestions and delays, in a fast way and with low overhead, in order to recommend alternative paths to other vehicles.

This paper is organized as follows. In Section 2 we address pre-existing different solutions for the broadcast storm problem in VANETs. Then, in Section 3 we introduce our proposed technique (i.e., SRB), whose aim is to mitigate the broadcast storm effect. Simulation results are presented in Section 4 . in order to validate our technique, by means of a comparison with traditional broadcast approach. We will show that SRB provides an automatic cluster detection mechanism, while keeping low the number of forwarder vehicles. Finally, conclusions are drawn in Section 5 


\section{Related Work}

In this section we give an overview of previous contributions in broadcasting protocols for VANETs, particularly focusing on cluster-based approaches. Within the discussion, we clarify the paper objective and then introduce our proposed approach.

The problem statement for reliable protocols is to design a protocol that can deliver a message from a single source to every node in her transmission range with the highest possible reliability and minimum delay. A successful message dissemination in VANETs needs an efficient decision mechanism in order to maximise reliability and keep the overhead low. The decision criterion about when and how a safety message should be delivered or repeated is an open issue.

Given the requirements of safety applications (i.e., low delay and effective reliability), and the limitations of vehicular communications (i.e, short-lived connectivity links), selective broadcast or multicast strategies seem more applicable than either unicast routing or flooding. In fact the latter generates a high overhead without increasing the success rate substantially, [6]. Several solutions have been made to introduce intelligence to the basic broadcast concept and make it more selective and, thus, more efficient in its resource usage.

A largely common assumption in connectivity models for VANETs is that a vehicular network is partitioned into a number of clusters [7]; vehicles within a partition can communicate either directly or through multiple hops among each other, but no direct connection exists between partitions, as depicted in Figure 1 A particular class of routing protocols uses this assumption by exploiting clusters formation, namely cluster-based approaches [8]. Each cluster has one cluster-head, responsible for intra and intercluster communications. In cluster-based routing protocols the formation of clusters and the selection of the cluster-head is an important issue that is very hard in VANETs due to the high mobility dynamic cluster formation process.

In [9] Ni et al. consider each cluster consists of three node types: head, gateway and member. The gateway nodes are those who connect to the gateway nodes in other clusters, while the cluster-head is a node whose transmission radius can reach everyone in the same cluster. Finally, members are those who do not belong in either head or gateway group. When a gateway node receives a message from other clusters, it will rebroadcast the message that will be received, and then further retransmitted, by the cluster-head. Although this cluster architecture is correct, the authors did not specify the procedure for the cluster-head election.

In [10] Fasolo et al. propose a Smart Broadcast protocol, which exploits vehicles' positions. The proposed technique assumes that the vehicular network is partitioned in adjacent sectors and that vehicles are able to estimate their own position and, therefore, the sector they belong to. The Smart Broadcast technique considers a contention resolution procedure to elect the relay nodes. Although this technique seems very efficient, it has not been validated in terms of network performance and system overhead. Another work which considers both information on vehicles' position and the cluster formation is presented by Luo et al. [11]. Their approach is a cluster-based routing protocol and the basic idea is to divide the geographic area into foursquare grids, where a vehicle is elected as the cluster-head to route data packets across nearby grids. Also this technique needs to be validated via simulation results.

In all previous works, mobility aspects have not been considered, while it is noticeable that the cluster selection process is particularly affected by vehicle mobility and cluster stability. In [12], Gunter et al. take into account mobility during cluster collision, and a cluster-head vehicle is that one with the lowest relative mobility and closest proximity to its neighbors. Alternatively, in [13] Kayis and Acarman classify nodes into speed groups, so that nodes belonging to the same speed group will be in the same cluster. Finally, a well-known mobility-based clustering technique is MOBIC [14], which considers an aggregate local mobility metric as the basis for cluster formation: the node with the smallest variance of relative mobility to its neighbors is elected as the cluster-head.

In this paper we propose $\mathrm{SRB}$, a reliable cluster-based routing protocol that is expected to minimize 


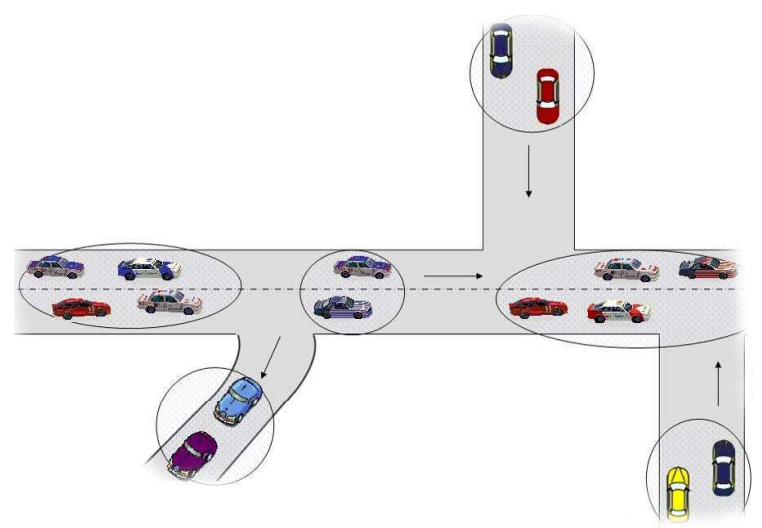

Figure 1: Disconnected vehicle clusters, due to gaps among consecutive clusters.

the number of rebroadcast messages. SRB considers the cluster selection process, and the cluster-head election, by exploiting the inter-vehicle distance and the time delay. Via simulation results, our technique results in an efficient method to detect clusters and alleviate the broadcast storm problem.

\section{Selective Reliable Broadcast}

The aim of SRB is twofold: $(i)$ to avoid rebroadcast messages (i.e., a limitation of broadcast storm problem), and (ii) to detect clusters in automatic and fast way. The proposed approach considers the message rebroadcast process within a VANET by selecting a limited number of vehicles, acting as forwarders. Far from traditional broadcast routing, SRB detects clusters of vehicles in a fast and efficient way, and elects one Cluster-Head $(\mathrm{CH})$ vehicle for each cluster detected. The $\mathrm{CH}$ is then selected as the next message forwarder.

Let us assume a hybrid traffic scenario, consisting of both high and low speed vehicles, as well as high and low vehicle density areas. This scenario well depicts a real vehicular environment, i.e. a clear highway with congested entries. To avoid sudden and emergency braking, information about congested areas should reach high speed approaching vehicles in a timely fashion.

We consider an aggregated vehicles group as a Zone-of-Relevance (ZOR) 1 , depicted by the following features i.e., (i) high vehicular density, and (ii) low vehicle speed. We will show how SRB is able to efficiently discover the ZOR (i.e., with low overhead and delay).

SRB leverages on two main assumptions, i.e. (i) the vehicular area is partitioned in adjacent sectors, and (ii) all vehicles equipped with GPS are able to estimate their own position. Network partitioning (or fragmentation, [7]) is introduced in order to accordingly propagate information messages inside the VANET, while avoiding multiple copies of the same message to the same recipients. Sectors provide loop-free message propagation along the transmission direction from the source vehicle.

SRB considers a contention resolution procedure necessary to detect the ZOR and nearby clusters, and then elects relay nodes, each per single cluster. The contention procedure and cluster detection mechanism are detailed as follows:

- RTB transmission: A source vehicle transmits an Request-to-Broadcast (RTB) control message to all neighboring vehicles in the transmission range. The RTB is a MAC-broadcast packet that contains the geographical position of the transmitter node;

\footnotetext{
${ }^{1}$ In this paper we use the term ZOR with the meaning of a congested traffic area.
} 


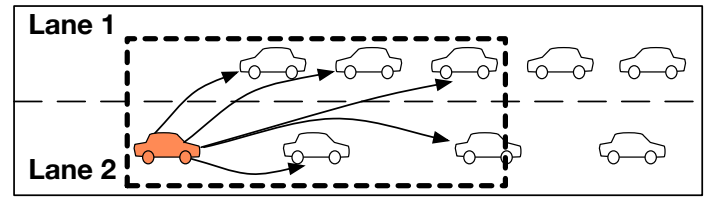

(a)

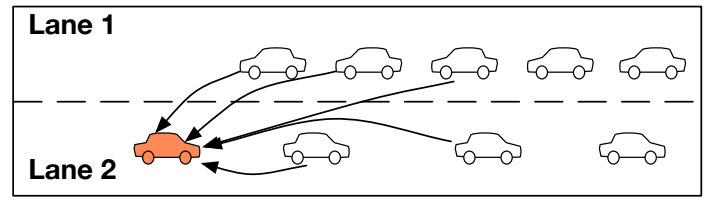

(b)

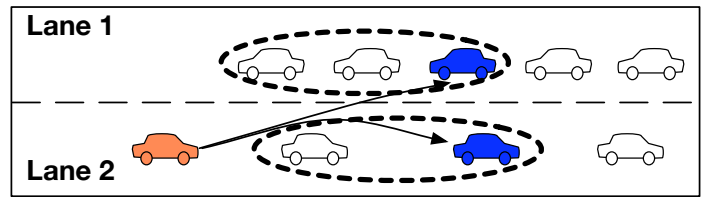

(c)

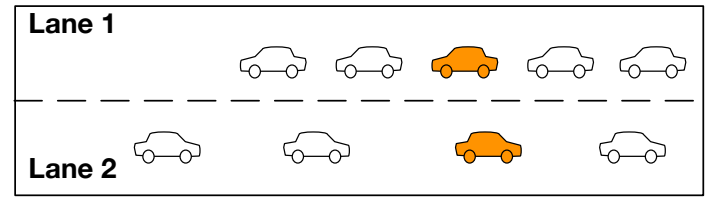

(d)

-1 Transmission range ك- Source/forwarder vehicle

:-- Cluster detected

Figure 2: Main phases of SRB technique: (a) RTB transmission, (b) CTB transmission, (c) Cluster detection and $\mathrm{CH}$ election, and $(d)$ message propagation.

- Waiting time calculation: Upon receiving an RTB, vehicles compute their distance from the source vehicle (i.e., $d[\mathrm{~m}])$. The distance information is exploited in the calculation of the waiting time (i.e., $t_{w}[\mathrm{~s}]$ ) - a backoff time - which depends on maximum and minimum contention window, as follows:

$$
t_{w}=\left[\frac{r_{t x}-d}{r_{t x}} \cdot\left(C W_{\max }-C W_{\min }\right)+C W_{\min }\right] \cdot t_{s l o t},
$$

where $r_{t x}[\mathrm{~m}]$ is the transmission range, and $C W_{\max , \min }$ are the maximum and minimum contention window sizes, respectively. By using (1) vehicles in the further regions always transmit before the others. According to the CSMA/CA policy of IEEE 802.11, the backoff time is decremented by 1 at each idle slot, while the decrease is stopped when the medium is busy;

- CTB transmission: Whenever the waiting time associated to a vehicle countdowns to zero, the vehicle sends back to the source a Clear-to-Broadcast (CTB) packet, containing the vehicle ID and its distance from the source. After receiving a valid CTB packet, vehicles exit the contention phase; in case of collisions, vehicles remain in the contention phase and resume the backoff process;

- Cluster detection: The source vehicle receives ID and distance from its nearby vehicles. By measuring the angle of arrival of the CTB messages, the source vehicle is able to calculate all the mutual inter-vehicle distances among its nearby vehicles. If the distance between each couple of nearby vehicles is lower than a threshold value (i.e., $D_{\min }$ ), the two vehicles will be considered belonging to the same cluster.

The choice of $D_{\min }$ influences the number of clusters identified: the higher the distance threshold, the higher the number of vehicles in each cluster. When $0 \leftarrow D_{\min }$, each vehicle identifies a 1-size cluster;

- Cluster-head election: After detecting multiple clusters, the source vehicle elects the furthest vehicle inside each cluster as the Cluster-Head, and transmits a data message only to such vehicle. 
Table 1: Message exchanged classification and content.

\begin{tabular}{c|c|c}
\hline \hline Message & Content & Size [byte] \\
\hline RTB & Source node's position & 7.5 \\
CTB & Source-Destination distance & 7.5 \\
Data & CH ID, data information & 1526 \\
\hline \hline
\end{tabular}

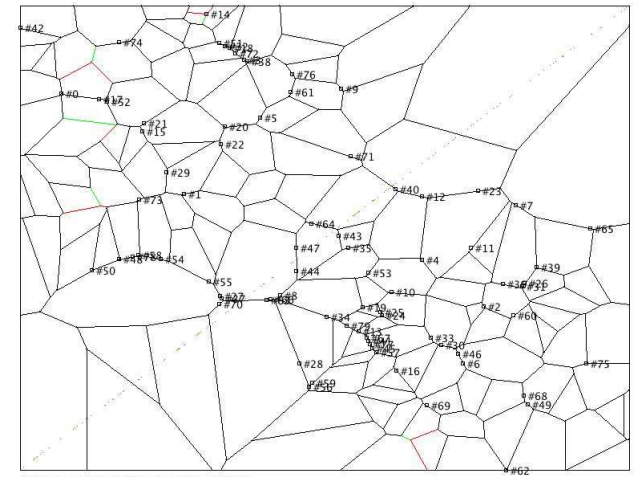

(a)

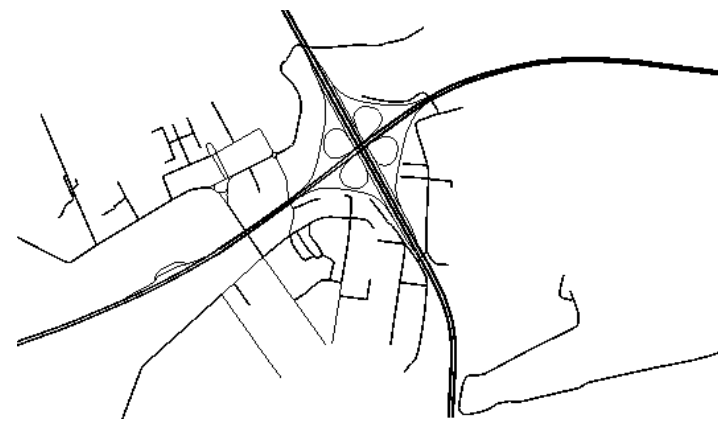

(b)

Figure 3: (a) Urban scenario generated with VanetMobiSim [15], and (b) highway scenario (i.e., a selected portion of G.R.A.) generated with SUMO [16].

Upon receiving the data message, each $\mathrm{CH}$ will become the message source for the next contention phase, and the SRB algorithm is repeated for the next hops. In Table 1 we report the main messages exchanged in the SRB technique. Figure 2 depicts the main phases of SRB technique, for a forward data transmission along the vehicular grid. Once the $\mathrm{CH}$ vehicles are identified, they will forward messages according to SRB protocol.

In the next performance evaluation section, we will show that our technique delivers data with reasonable delay to most of the intended vehicles. Moreover, the amount of transmitted packets per hop depends on the number of selected vehicles as forwarders, and it is expressed as:

$$
p k t / h o p=\sum_{i=1}^{N}\left(2 n_{i}+1\right),
$$

where $N$ is the number of clusters detected by a source node per hop, and $n_{i}$ is the number of vehicles comprising the $i$-th cluster. The terms $2 n_{i}$ and 1 represent respectively the maximum number of packets RTB and CTB, and one data packet, transmitted in each hop $2^{2}$

\footnotetext{
${ }^{2} \mathrm{Eq}$. 2. represents an ideal scenario where drop packets and any collisions are omitted, or at least minimized.
} 

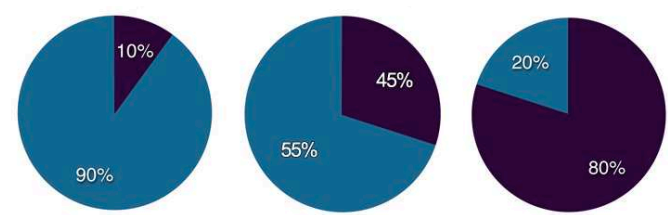

Figure 4: Percentage of selected $\mathrm{CH}$ vehicles (dark blue) for different values of distance threshold. From left to right, $D_{\min }=250 \mathrm{~m}, 150 \mathrm{~m}$, and $50 \mathrm{~m}$. Rest of vehicles (light blue) is not involved into message dissemination process.

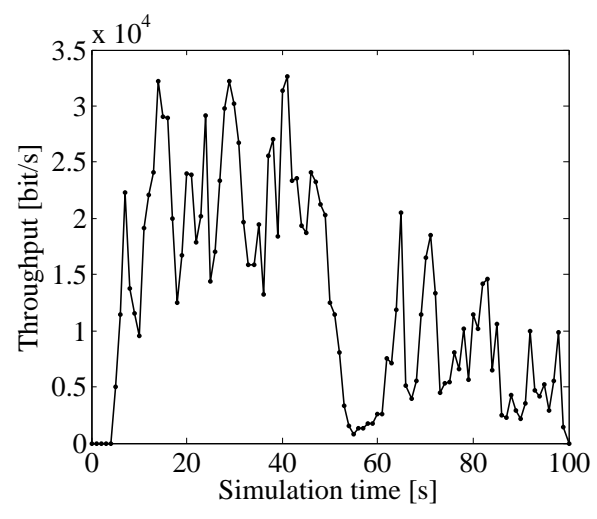

Figure 5: Urban scenario. Average instant throughput for SRB technique vs. simulation time.

\section{Simulation Results}

In this section, we analyse the performance of the proposed technique in two different cases i.e., (i) urban, and (ii) highway scenario. For urban scenario, we generate a 4 kilometers square grid with one lane per direction. In highway scenario, we select a 4 kilometers square area comprised of straight roads with 3 lanes per direction and several entries.

In both scenarios, we assume there are some ZORs, as previously defined, in unknown areas. Packets are generated with a constant generation rate (i.e., $\lambda[\mathrm{pck} / \mathrm{s}]$ ), and are transmitted according to a fixed data rate (i.e., $R[\mathrm{Mb} / \mathrm{s}]$ ), within a fixed transmission range (i.e., $\left.r_{t x}[\mathrm{~m}]\right)$. All vehicles are equipped with a GPS receiver and radio interfaces, compliant with the IEEE $802.11 b$ standard, with the following characteristics: $r_{t x}=300 \mathrm{~m}$ and $R=6$ Mbps.

\subsection{Urban scenario}

In this case, we adopt the VanetMobiSim software [15] to generate a 4 kilometers square grid, as shown in Figure $3(a)$. Performance have been evaluated in terms of $(i)$ throughput, (ii) number of elected CHs, (iii) message propagation, and (iv) end-to-end delay, by means of numerical simulations carried out with the ns-2.34 simulator [17]. In this scenario, we assume 150 vehicles are moving at constant speeds, and forming clusters only for a limited — short-life — time interval.

The SRB performance mainly depends on the value of the distance threshold $D_{\min }$, since this parameter affects the number of vehicles in a cluster. As a consequence, the higher the distance threshold, the lower the number of clusters detected, or equally the number of $\mathrm{CH}$ vehicles. Figure 4 depicts the percentage of CHs for different values of $D_{\min }$. Notice that for a low value of distance threshold the SRB is not 


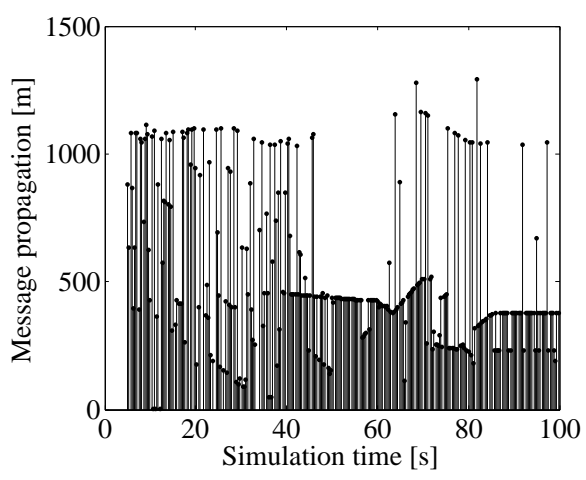

Figure 6: Urban scenario. Average message propagation for SRB technique vs. simulation time.

able to select a limited number of $\mathrm{CHs,} \mathrm{and} \mathrm{approximates} \mathrm{the} \mathrm{behavior} \mathrm{of} \mathrm{a} \mathrm{traditional} \mathrm{broadcast} \mathrm{protocol}$ (i.e., all neighboring vehicles are involved in data forwarding). In our simulations, we have considered $D_{\min }=150 \mathrm{~m}$, which represents a good tradeoff with the transmission range (i.e., $D_{\min }=r_{t x} / 2$ ).

Figure 5] depicts the average throughput [bit/s] experienced by vehicles communicating via SRB in the vehicular scenario during the simulation time. During the simulation, the vehicle cluster aggregation occurs in a random fashion, due to not homogeneous nature of the urban scenario (i.e., presence of junctions and traffic lights can reduce cluster formation). Leveraging on this consideration, we can observe a variable behavior of throughput: in the first part of the simulation (i.e., for $t<50 \mathrm{~s}$ ), the throughput shows high values and reaches $30 \mathrm{kbps}$, while in the second part (i.e., for $t \geq 50 \mathrm{~s}$ ) low values of throughput occur. This is justified by an increase of data exchange which occurs for $t<50 \mathrm{~s}$, when the cluster detection occurs; while for $t \geq 50 \mathrm{~s}$ vehicles aggregation in clusters is reduced and traffic flow becomes uncongested.

This result can be better analysed together with the number of elected $\mathrm{CH}$ vehicles, giving information about the effectiveness of the proposed protocol. Compared to traditional broadcast protocols, the SRB selects the $45 \%$ of vehicles as $\mathrm{CHs}$ and reaches an average throughput of $13 \mathrm{kbps}$. The resource usage is then limited.

In Figure 6 is depicted the average message propagation [m] during the simulation time. Data messages propagate in the network and reach on average long distances (i.e., up to $1.3 \mathrm{~km}$ far from the source). The vehicular environment is then almost fully "covered". It is also important to notice that performance trend reaches high values since a few seconds from the beginning of the simulation. These results suggest that the vehicular connectivity is largely guaranteed in the network. Finally, in Figure 7 we show the average end-to-end delay [s], versus the simulation time. We can notice a delay on average lower than $400 \mathrm{~ms}$, which is particularly suitable for safety applications. Some delay peaks occur, which are due to multi-hop message propagation time, and the waiting time parameter, while the processing time to select $\mathrm{CHs}$ has been considered negligible.

Previous simulation results have show that in urban scenarios, where vehicle density is not uniform and can limit the cluster formation, SRB provides high message propagation distance with low end-to-end delay, as well as an effective throughput. In next Subsection 4.2, we will show the benefits of SRB are better highlighted in highway scenario. 


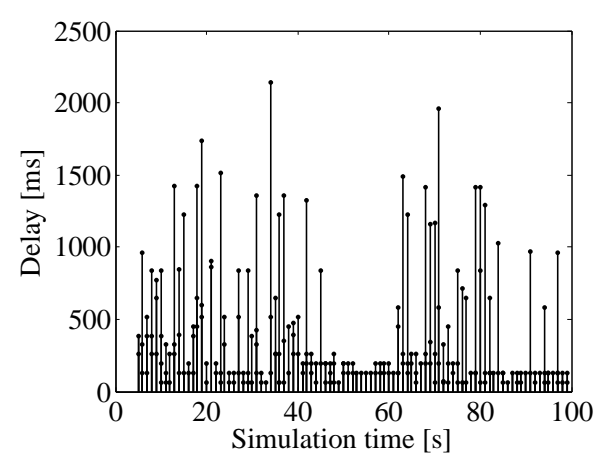

Figure 7: Urban scenario. Average end-to-end delay for SRB technique vs. simulation time.

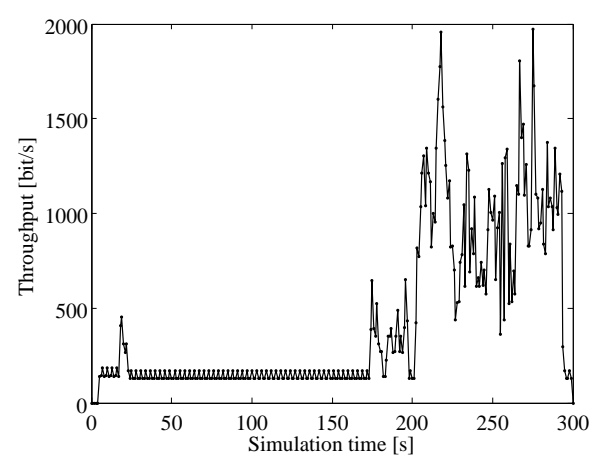

Figure 8: Highway scenario. Average throughput for SRB technique vs. simulation time. Cluster detection occurs for $t \geq 175 \mathrm{~s}$, as highlighted by means of an increase of throughput.

\subsection{Highway scenario}

The second scenario, shown in Figure 3 (b), is based on a real urban map of a square-shaped portion of the city of Rome (Italy), with an area equal to $15 \mathrm{~km}^{2}$. The map has been retrieved from the website of the Open Street Map (OSM) [18]. The scenario represents a portion of GRA (Grande Raccordo Anulare, literally "Great Ring Road"), that is a toll-free, ring-shaped orbital motorway encircling Rome. GRA is one of the most important roads in Rome, and traffic reaches up to 160.000 vehicles per day. GRA features up to 42 junctions and in rush hours traffic congestions affect the road condition.

In such scenario we validated the SRB technique works very efficiently to detect clusters in an automatic and fast way, so that vehicles can avoid traffic congestions, and potentially are rerouted to different paths. The simulated scenario consists of a congested area with at least 15 vehicles driving in a platoon at low speed, while 70 vehicles drive into a nearby free area at high speed. A vehicle coming from the free area should be able to fast detect the traffic congestion when approaching. Unlikely the urban scenario where vehicles form clusters randomly, in highway environment we consider a huge cluster in a limited area, depicted by low vehicle speed and high vehicle density. The simulations have used a traffic simulator called Simulation of Urban Mobility (SUMO) [16] to generate traffic scenarios, and the trace files have been simulated on the network simulation.

In this section we describe the most significant results, which allow to detect clusters. Particularly, $(i)$ the average throughput, and (ii) the Packet Delivery Ratio (PDR), that is the ratio between the amount of delivered data messages and all delivered messages. 


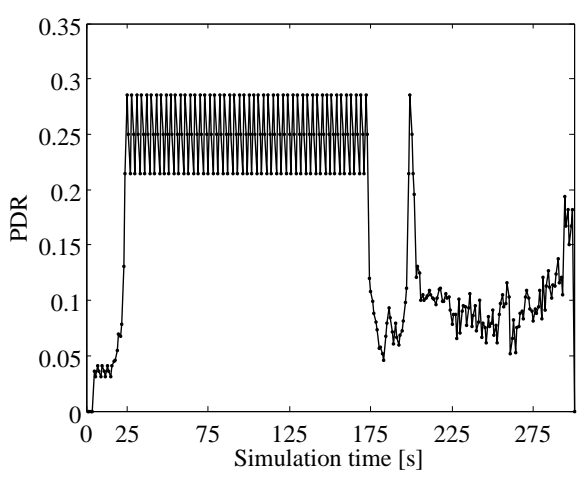

Figure 9: Highway scenario. Average PDR for SRB technique vs. simulation time. Notice the reduction of PDR due to the cluster detection process.

Figure 8 shows the average throughput vs. the simulation time. We notice a high increase of throughput for $t \geq 175 \mathrm{~s}$, when it reaches up to $1.8 \mathrm{kbps}$. This behavior is justified since at $t=175 \mathrm{~s}$ a vehicle moving in the highway approaches the congested area, and then an high number of data messages are exchanged. However, due to the nature of highway scenario, with a huge cluster of vehicles, the average value of throughput is lower than that in urban scenario.

Finally, the effectiveness of SRB has been also evaluated in terms of PDR, which gives information about the percentage of delivered data messages. It is defined as

$$
P D R=\frac{p k t_{d a t a}}{p k t_{R T B}+p k t_{C T B}+p k t_{d a t a}},
$$

where $p k t_{R T B, C T B}$ is respectively the number of RTB and CTB messages exchanged, while $p k t_{d a t a}$ is the number of delivered data messages.

Figure 9 depicts the PDR trend versus the simulation time. Again, for $t<175 \mathrm{~s}$ the PDR shows fluctuations around 0.25 , while for $t \geq 175 \mathrm{~s}$ the trend has on average low values around 0.1 . This is justified by the increase of RTB and CTB messages, which are exchanged during the cluster detection process. 


\section{Conclusions}

In this paper we presented a novel reliable broadcast protocol that selectively transmits messages within a vehicular ad-hoc network. The proposed approach, namely SRB, aims to alleviate the broadcast storm problem, which strongly affects the network overload. SRB is particularly effective for safety applications: it relies on cluster-based routing protocols, as well as exploits the vehicles' positions, in order to detect traffic congestions (i.e., car platoons) in a fast way and with low overhead. Only a limited number of vehicles are elected as cluster-heads to forward messages. This allows to save network resources while keeping high performance.

SRB has been validated through extensive simulations, both in urban and highway scenarios. Results have shown that the proposed approach is able to detect vehicle clusters, and provides high distances for message dissemination, in respect of traditional broadcast protocols. Especially in highway scenarios, SRB works efficiently by means of detecting congestion areas in a fast way.

Future work will be address to extend the use of SRB protocol to context-aware applications, where vehicles should be able to forward advertisement messages only to vehicles in a selected area.

\section{References}

[1] H. Hartenstein and K. Laberteaux, VANET: Vehicular Applications and Inter-networking Technologies. Wiley \& sons, Ltd., 2010.

[2] O. Tonguz, N. Wisitpongphan, F. Bai, P. Mudalige, and V. Sadekar, "Broadcasting in VANET," in Proc. of Mobile Networking for Vehicular Environments, pp. 7-12, May 2007.

[3] N. Wisitpongphan, O. Tonguz, J. Parikh, P. Mudalige, F. Bai, and V. Sadekar, "Broadcast storm mitigation techniques in vehicular ad hoc networks," IEEE Wireless Communications, vol. 14, pp. 8494, December 2007.

[4] K. Bür and M. Kihl, "Evaluation of Selective Broadcast Algorithms for Safety Applications in Vehicular Ad Hoc Networks," International Journal of Vehicular Technology, vol. 2011, p. 13, 2011.

[5] O.K.Tonguz, N. Wisitpongphan, J. Parikh, F. Bai, P. Mudalige, and V. Sadekar, "On the Broadcast Storm Problem in Ad hoc Wireless Networks," in Proc. of 3rd International Conference on Broadband Communications, Networks and Systems, BROADNETS, pp. 1-11, October 2006.

[6] W. Chen, R. K. Guha, T. J. Kwon, J. Lee, and I. Y. Hsu, "A survey and challenges in routing and data dissemination in vehicular ad-hoc networks," in Proc. of IEEE International Conference on Vehicular Electronics and Safety, (Columbus, Ohio, USA), pp. 328-333, 2008.

[7] T. Little and A. Agarwal, “An Information Propagation Scheme for Vehicular Networks," in Proc. of IEEE Intelligent Transportation Systems Conference, (Vienna, Austria), pp. 155-160, 2005.

[8] J. Kakarla, S. Sathya, G. B. Laxmiand, and R. B. Babu, "A Survey on Routing Protocols and its Issues in VANET," International Journal of Computer Applications, vol. 28, pp. 38-44, August 2011.

[9] S. Ni, Y. Tseng, Y. Chen, and J. Sheu, "The broadcast storm problem in a mobile ad hoc network," in Proc. of the 5 th annual ACM/IEEE Intl. Conf. on Mobile Computing and Networking (MobiCom), pp. 151-162, 1999. 
[10] E. Fasolo, A. Zanella, and M. Zorzi, “An Effective Broadcast Scheme for Alert Message Propagation in Vehicular Ad hoc Networks," in Proc. of IEEE International Conference on Communications (ICC), vol. 9, pp. 3960-3965, 2006.

[11] Y. Luo, W. Zhang, and Y. Hu, "A New Cluster Based Routing Protocol for VANET," in Proc. on 2nd Intl. Conf. on Networks Security Wireless Communications and Trusted Computing (NSWCTC), vol. 1, pp. 176-180, 24-25 April 2010.

[12] Y. Gunter, B. Wiegel, and H. Grossmann, "Cluster-based Medium Access Scheme for Vanets," in Proc. on Intelligent Transportation Systems Conference, 2007.

[13] O. Kayis and T. Acarman, "Clustering formation for inter-vehicle communication," in Proc. on IEEE Intelligent Transportation Systems Conference (ITST), pp. 636-641, October 2007.

[14] P. Basu, N. Khan, and T. Little, "A mobility based metric for clustering in mobile ad hoc networks," in Proc. on International Conference on Distributed Computing Systems Workshop, pp. 413-418, April 2001.

[15] "VanetMobiSim.” Website: http://vanet.eurecom.fr/.

[16] “SUMO project.” Website: http://sourceforge.net/projects/sumo/.

[17] "Network simulator 2 (ns-2)." available at: http://isi.edu/nsnam/ns/.

[18] “Open street map.” website: http://www.openstreetmap.org. 


\section{Contents}

\begin{tabular}{llr}
\hline & Introduction & 3
\end{tabular}

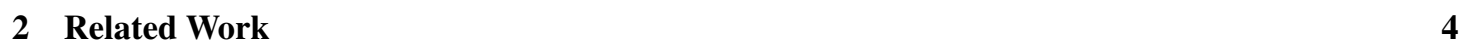

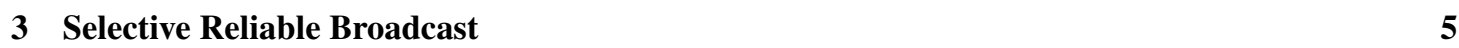

\begin{tabular}{llr}
4 Simulation Results & 8 \\
\hline
\end{tabular}

4.1 Urban scenario . . . . . . . . . . . . . . . . . . . . . . . . 8

4.2 Highway scenario $\ldots \ldots \ldots \ldots \ldots \ldots \ldots$

\begin{tabular}{llr}
\hline Conclusions & 12
\end{tabular} 


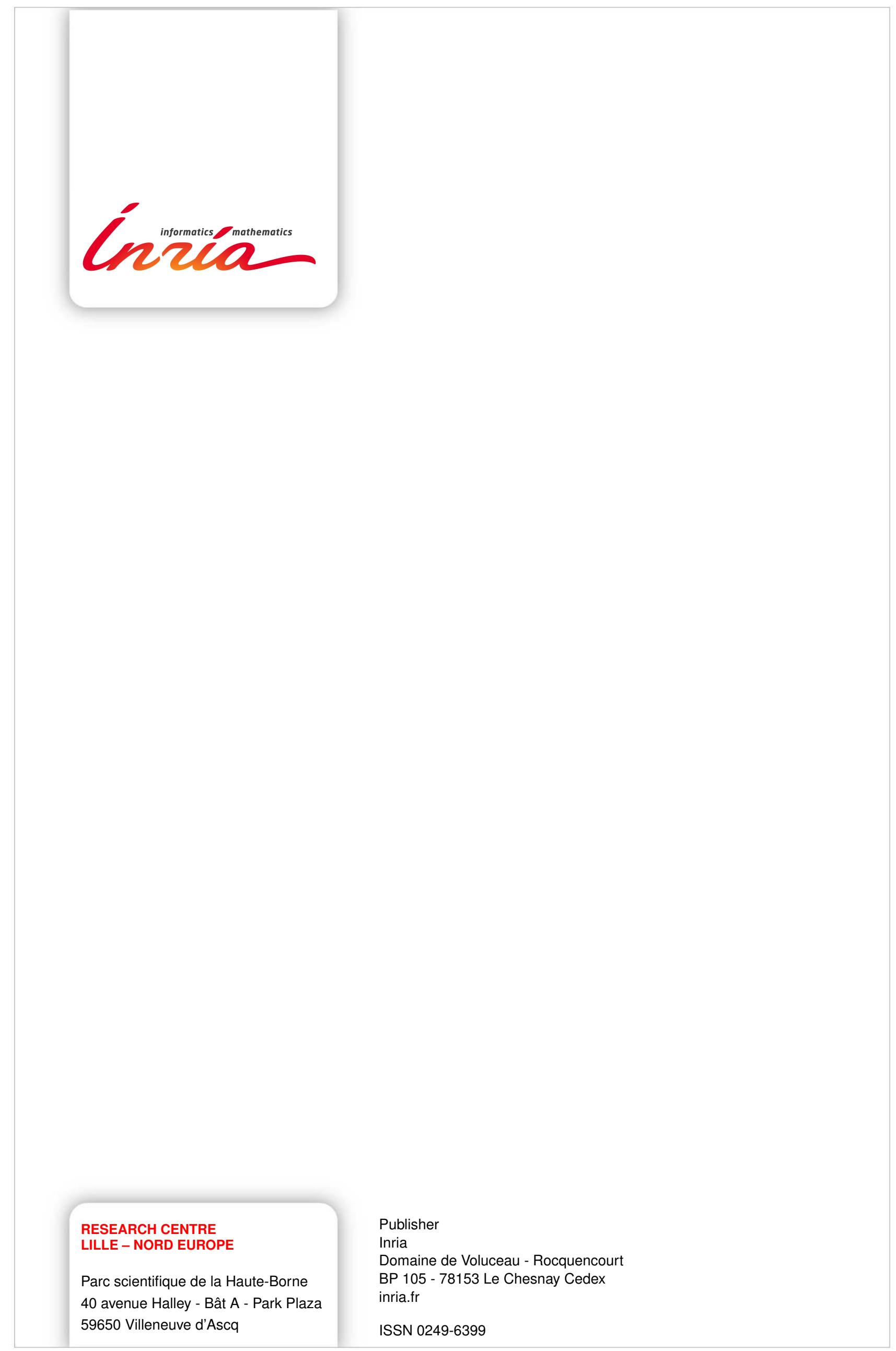

